\title{
Guest Editorial: Special Issue on Matching Under Preferences
}

\author{
David F. Manlove · Robert W. Irving • Kazuo Iwama
}

Received: 14 May 2010 / Accepted: 16 May 2010 / Published online: 25 May 2010

(C) Springer Science+Business Media, LLC 2010

Matching problems with preferences occur in widespread applications such as the assignment of school-leavers to universities, junior doctors to hospitals, students to campus housing, children to schools, kidney transplant patients to donors and so on. The common thread is that agents have preference lists over the possible outcomes and the task is to find a matching of the participants that is in some sense optimal with respect to these preferences.

The archetypal problem of this kind is the celebrated Stable Marriage problem, first introduced by David Gale and Lloyd Shapley in 1962. Over the last decade there has been something of a resurgence of interest in the algorithmic study of variants of this problem. In addition, research has been instigated into related matching problems, for example bipartite models with one-sided preferences, under criteria such as rank-maximality, popularity and Pareto optimality. These developments can be attributed in part to the growing tendency for matching processes to involve the online collection of preference information, necessitating efficient algorithms for constructing matchings that are fair and optimal in an expanding range of problem variants.

Dedicated to the memory of David Gale, 13 December 1921-7 March 2008.

D.F. Manlove $(\bowtie) \cdot R$.W. Irving

Department of Computing Science, University of Glasgow, Glasgow G12 8QQ, UK

e-mail: davidm@dcs.gla.ac.uk

R.W. Irving

e-mail: rwi@dcs.gla.ac.uk

K. Iwama

School of Informatics, Kyoto University, Kyoto 606-8501, Japan

e-mail: iwama@kuis.kyoto-u.ac.jp 
The time is right, therefore, for a special issue of a leading journal to focus on matching problems with preferences, with particular emphasis on the algorithms and complexity perspective. We solicited papers studying matching problems including (but not limited to) bipartite matching problems with preference lists on both sides (such as the Stable Marriage or Hospitals/Residents problems), bipartite matching problems with one-sided preferences (such as house allocation or paper assignment problems) and non-bipartite matching problems involving preferences (such as the Stable Roommates problem), under optimality criteria such as stability, rankmaximality, popularity and Pareto optimality.

This special issue includes selected papers from the workshop "Match-UP: Matching Under Preferences-Algorithms and Complexity" that were invited for submission to this issue of Algorithmica. The Match-UP workshop was held in Reykjavík on 6 July 2008 as a satellite workshop of ICALP 2008 and was very well attended, attracting excellent invited and contributed talks. The opening talk at the workshop was to have been given by one of the key pioneers in the field, Professor David Gale, of the University of California, Berkeley. Sadly, David Gale died suddenly on 7 March 2008, while preparations for the workshop were under way. We are honoured to dedicate this special issue to his memory.

Six papers that are fully revised and extended versions of earlier submissions to the Match-UP workshop were invited for submission to this special issue. In addition, the remaining six papers that appear here were submitted in response to a general call for papers that was issued shortly after the workshop (and not restricted to authors who had participated in the Match-UP workshop). All submissions were fully refereed to the usual standards of Algorithmica. We were delighted with the response (in terms of both quality and quantity) to both our paper invitations and the more general call for submissions, and feel that the twelve papers appearing in this special issue constitute an excellent snapshot of the current state of the art regarding research in the field.

A brief summary of the twelve papers appearing in this special issue follows.

- In "Three-Sided Stable Matchings with Cyclic Preferences", Péter Biró and Eric McDermid consider the three-sided extension of the Stable Marriage problem (featuring men, women and dogs) in which preference lists are cyclic in nature, and show that it is NP-complete to determine whether a stable matching exists, for two stability criteria.

- Housing markets in which some agents' houses are equivalent are considered by Katarína Cechlárová and Tamás Fleiner in "Housing Markets Through Graphs". They show that, in this context, determining whether an economic equilibrium exists is polynomial-time solvable for strict preferences, but NP-complete for socalled trichotomous preferences.

- Christine T. Cheng studies the complexity of finding generalized median stable matchings in instances of the Stable Marriage problem in "Understanding the Generalized Median Stable Matchings". She contributes polynomial-time algorithms, NP-hardness, \#P-hardness and approximability results for problems arising in this context.

- In "An Efficient Algorithm for Batch Stability Testing", John Dabney and Brian C. Dean answer an open question of Gusfield and Irving [1] by demonstrating that a set of matchings in an instance of the Stable Marriage problem with $n$ men and $n$ 
women can be checked for stability in time sub-quadratic in $n$ (following sufficient pre-processing).

- Brian C. Dean and Siddharth Munshi study many-many variants of the Stable Marriage and Stable Roommates problems in "Faster algorithms for Stable Allocation Problems". They give strongly polynomial-time algorithms for finding stable allocations in both the bipartite and non-bipartite settings, and also extend their results to the problem of finding optimal stable allocations.

- Another variant of the Stable Roommates problem, this time where agents have substitutable choice functions, is considered by Tamás Fleiner in "The Stable Roommates Problem with Choice Functions". He shows that the problem of finding a stable matching, or reporting that none exists, is polynomial-time solvable for so-called increasing substitutable choice functions, though NP-hard for general substitutable choice functions.

- Patrik Floréen, Petteri Kaski, Valentin Polishchuk and Jukka Suomela consider distributed algorithms for finding matchings with few blocking pairs in instances of the Stable Marriage problem in their paper "Almost Stable Matchings by Truncating the Gale-Shapley Algorithm".

- In "Assigning Papers to Referees", Naveen Garg, Telikepalli Kavitha, Amit Kumar, Kurt Mehlhorn and Julián Mestre study precisely this in the context of conference paper review assignment. They formulate a notion of a fair assignment, and give polynomial-time algorithms, NP-hardness results and approximation algorithms for problems involving computing such matchings.

- "Circular Stable Matching and 3-way Kidney Transplant" by Chien-Chung Huang again studies the three-sided variant of the Stable Marriage problem and complements nicely the aforementioned paper of Péter Biró and Eric McDermid. The author also considers cyclic preferences but demonstrates NP-hardness results for different cases of the problem, and, in addition, contributes structural and \#P-hardness results.

- Hirotatsu Kobayashi and Tomomi Matsui study strategic issues in the Stable Marriage problem in "Cheating Strategies for Gale-Shapley Algorithm with Complete Preference Lists". In particular, given a complete set of men's preferences and a partial matching $M$ of men to women, the authors present algorithmic results for the problem of finding a set of women's preferences (if they exist) such that the man-oriented Gale-Shapley algorithm returns a stable matching that contains $M$.

- The Stable Marriage problem is again considered by Dániel Marx and Ildikó Schlotter, this time where preference lists may be incomplete and may include ties, in "Parameterized Complexity and Local Search Approaches for the Stable Marriage Problem with Ties". The authors present parameterized complexity results for the problems of computing maximum size, egalitarian and minimum regret stable matchings in this context.

- In "A Polynomial-Time Algorithm to find von Neumann-Morgenstern Stable Matchings in Marriage Games", Jun Wako gives an $O\left(n^{2}\right)$ algorithm for finding a man-optimal matching and a woman-optimal matching in a von NeumannMorgenstern stable set for a given instance of the Stable Marriage problem with $n$ men and $n$ women.

The guest editors would like to thank all of the contributors for their work. We would also like to thank the referees for their detailed and timely reviews. In addition, 
we thank Ming-Yang Kao, Editor-in-Chief of Algorithmica, for suggesting the special issue and for his support and advice during the editorial process. Finally we would like to thank the editorial team at Springer for their administrative support throughout.

\section{References}

1. Gusfield, D., Irving, R.W.: The Stable Marriage Problem: Structure and Algorithms. MIT Press, Cambridge (1989) 